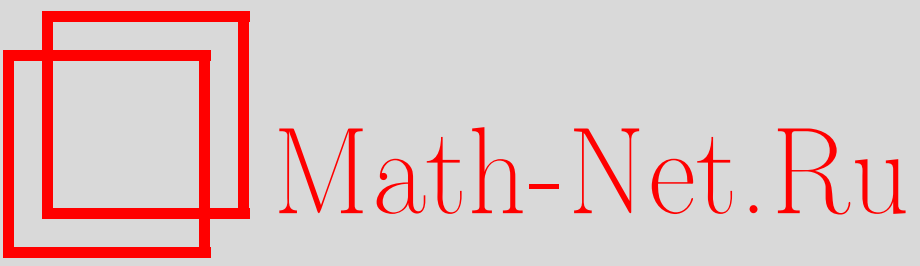

О. И. Мохов, Согласованные и почти согласованные метрики, УМH, 2000, том 55, выпуск 4, 217-218

DOI: https://doi.org/10.4213/rm318

Использование Общероссийского математического портала Math-Net.Ru подразумевает, что вы прочитали и согласны с пользовательским соглашением

http://www.mathnet.ru/rus/agreement

Параметры загрузки:

IP : 54.198 .64 .247

26 апреля 2023 г., 12:59:17 


\title{
СОГЛАСОВАННЫЕ И ПОЧТИ СОГЛАСОВАННЫЕ МЕТРИКИ
}

\author{
О. И. Мохов
}

В заметке вводятся понятия согласованных и почти согласованных псевдоримановых метрик, обобщающие понятие плоского пучка метрик [1] (см. также [2]-[4]). Согласованные метрики порождают согласованные пуассоновы структуры гидродинамического типа (эти структуры являются локальными для плоских метрик [5] и нелокальными, если метрики - неплоские [6]-[8], [3]).

ОпредЕлениЕ 1. Две псевдоримановы контравариантные метрики $g_{1}^{i j}(u)$ и $g_{2}^{i j}(u)$ называются согласованными, если для любой линейной комбинации этих метрик

$$
g^{i j}(u)=\lambda_{1} g_{1}^{i j}(u)+\lambda_{2} g_{2}^{i j}(u),
$$

где $\lambda_{1}$ и $\lambda_{2}$-произвольные константы, для которых $\operatorname{det}\left(g^{i j}(u)\right) \neq 0$, коэффициенты соответствующих связностей Леви-Чивита и компоненты соответствующих тензоров римановой кривизны связаны тем же самым линейным соотношением:

$$
\begin{aligned}
& \Gamma_{k}^{i j}(u)=\lambda_{1} \Gamma_{1, k}^{i j}(u)+\lambda_{2} \Gamma_{2, k}^{i j}(u), \\
& R_{k l}^{i j}(u)=\lambda_{1} R_{1, k l}^{i j}(u)+\lambda_{2} R_{2, k l}^{i j}(u) .
\end{aligned}
$$

Мы также будем говорить в этом случае, что метрики $g_{1}^{i j}(u)$ и $g_{2}^{i j}(u)$ образуют пучок метрик.

ОпредЕлЕниЕ 2. Две псевдоримановы контравариантные метрики $g_{1}^{i j}(u)$ и $g_{2}^{i j}(u)$ называются почти согласованными, если для любой линейной комбинации таких метрик (1) выполняется условие (2).

ОПРЕдЕЛЕНИЕ 3. Две псевдоримановы метрики $g_{1}^{i j}(u)$ и $g_{2}^{i j}(u)$ называются неособой napoй метрик, если собственные значения этой пары метрик, то есть корни уравнения

$$
\operatorname{det}\left(g_{1}^{i j}(u)-\lambda g_{2}^{i j}(u)\right)=0
$$

различны.

В случае, когда метрики $g_{1}^{i j}(u)$ и $g_{2}^{i j}(u)$ - плоские, то есть $R_{1, j k l}^{i}(u)=R_{2, j k l}^{i}(u)=0$, соотношение (3) превращается в условие, что произвольная линейная комбинация плоских метрик $g_{1}^{i j}(u)$ и $g_{2}^{i j}(u)$ тоже всегда является плоской метрикой, и определение 1 эквивалентно известному определению плоского пучка метрик или, другими словами, согласованной пары локальных невырожденных пуассоновых структур гидродинамического типа [1] (см. также [2]-[4]).

В 1995 году в [3] Ферапонтов предложил подход к задаче о плоских пучках метрик, мотивированный теорией операторов рекурсии, и сформулировал критерий согласованности локальных невырожденных пуассоновых структур гидродинамического типа: тензор Нейенхейса аффинора, задаваемого метриками, тождественно равняется нулю. К сожалению, в общем случае этот критерий неверен. Из обнуления соответствуюшего тензора Нейенхейса, вообще говоря, не следует согласованность плоских метрик. Мы приведем далее соответствующие контрпримеры. В общем случае, как будет показано ниже, равенство нулю соответствующего тензора Нейенхейса является в действительности критерием почти согласованности плоских метрик, что не гарантирует согласованность соответствующих локальных пуассоновых структур гидродинамического типа. Критерий, сформулированный Ферапонтовым в [3], верен для любой неособой пары плоских метрик, и, более того, он допускает существенное обобщение на случай любой неособой пары псевдоримановых метрик, в частности, на особенно важные в теории систем гидродинамического типа случаи метрик постоянной римановой кривизны или метрик, порождающих общие нелокальные пуассоновы структуры гидродинамического типа.

Работа выполнена при финансовой поддержке Фонда Александра фон Гумбольдта (Германия), а также РФФИ (гранты №o 99-01-00010 и 96-15-96027) и INTAS (грант № 96-0770). 
ТеОРема 1. Если для произвольной линейной комбинации (1) двух метрик $g_{1}^{i j}(u)$ и $g_{2}^{i j}(u)$ выполняется условие (2), то тензор Нейенхейса аффинора $v_{j}^{i}(u)=g_{1}^{i s}(u) g_{2, s j}(u)$ тождественно равен нулю. Если пара метрик $g_{1}^{i j}(u)$ и $g_{2}^{i j}(u)$ является неособой, то из обнуления тензора Нейенхейса аффинора $v_{j}^{i}(u)=g_{1}^{i s}(u) g_{2, s j}(u)$ следует согласованность метрик $g_{1}^{i j}(u)$ и $g_{2}^{i j}(u)$.

Введем тензор

(5) $\quad M^{i j k}(u)=g_{1}^{i s}(u) \Gamma_{2, s}^{j k}(u)-g_{2}^{j s}(u) \Gamma_{1, s}^{i k}(u)-g_{1}^{j s}(u) \Gamma_{2, s}^{i k}(u)+g_{2}^{i s}(u) \Gamma_{1, s}^{j k}(u)$.

Лемма 1. Тензор $M^{i j k}(u)$ тождественно равен нулю тогда и только тогда, когда метрики $g_{1}^{i j}(u)$ и $g_{2}^{i j}(u)$ являются почти согласованньми.

Введем аффинор $v_{j}^{i}(u)=g_{1}^{i s}(u) g_{2, s j}(u)$ и рассмотрим тензор Нейенхейса этого аффинора

$$
N_{i j}^{k}(u)=v_{i}^{s}(u) \frac{\partial v_{j}^{k}}{\partial u^{s}}-v_{j}^{s}(u) \frac{\partial v_{i}^{k}}{\partial u^{s}}+v_{s}^{k}(u) \frac{\partial v_{i}^{s}}{\partial u^{j}}-v_{s}^{k} \frac{\partial v_{j}^{s}}{\partial u^{i}}
$$

следуя [3], где аналогично рассматривался аффинор $v_{j}^{i}(u)$ и его тензор Нейенхейса для двух плоских метрик.

ТеОрема 2. Метрики $g_{1}^{i j}(u)$ и $g_{2}^{i j}(u)$ являются почти согласованньци тогда и только тогда, когда соответствующий тензор Нейенхейса $N_{i j}^{k}(u)(6)$ тождественно равен нулю.

Лемма 2. Всегда имеют место следующие тождества:

(7) $\quad g_{1, s p}(u) N_{r q}^{p}(u) g_{2}^{r j}(u) g_{2}^{q i}(u) g_{2}^{s k}(u)=M^{k i j}(u)+M^{j k i}(u)+M^{j i k}(u)$,

(8) $2 M^{k i j}(u)=g_{1, s p}(u) N_{r q}^{p}(u) g_{2}^{r j}(u) g_{2}^{q i}(u) g_{2}^{s k}(u)-g_{1, s p}(u) N_{r q}^{p}(u) g_{2}^{r j}(u) g_{2}^{q k}(u) g_{2}^{s i}(u)$.

Теорема 3. Произвольная неособая пара метрик является согласованной тогда и только тогда, когда существуют локальнье координаты $u=\left(u^{1}, \ldots, u^{N}\right)$, такие что $g_{2}^{i j}(u)=g^{i}(u) \delta^{i j}$ u $g_{1}^{i j}(u)=f^{i}\left(u^{i}\right) g^{i}(u) \delta^{i j}$, где $f^{i}\left(u^{i}\right), i=1, \ldots, N,-$ произвольнье функиии одной переменной.

Плоские метрики $g^{i j}(u)=\exp \left(u^{1} u^{2}\right) \delta^{i j}, 1 \leqslant i, j \leqslant 2$, и $g_{2}^{i j}(u)=\delta^{i j}, 1 \leqslant i, j \leqslant 2$, являются почти согласованными, тензор Нейенхейса (6) для них тождественно равен нулю. При этом согласованными эти метрики не являются, и их сумма не является плоской метрикой.

ПреДЛОЖенИЕ. Любая непостоянная вещественная гармоническая функция $а(u)$ определяет пару почти согласованных плоских метрик $g^{i j}(u)=\exp (a(u)) \delta^{i j}, 1 \leqslant i, j \leqslant 2$, и $g_{2}^{i j}(u)=\delta^{i j}, 1 \leqslant i, j \leqslant 2$, которые не являются согласованными.

\section{СПИСОК ЛИТЕРАТУРЫ}

[1] Dubrovin B. // Lecture Notes in Math. 1996. V. 1620. P. 120-348; hep-th/9407018. [2] Dubrovin B. // Preprint SISSA 25/98/FM; math.DG/9803106. [3] Ferapontov E. V. // Topics in topology and mathematical physics / ed. S. P. Novikov. Providence, RI: Amer. Math. Soc., 1995. Р. 33-58. [4] Мохов О. И. // Труды МИАН. 1999. Т. 225. С. 284-300. [5] Дубровин Б. А., Новиков С. П. // Докл. АН СССР. 1983. Т. 270. № 4. С. 781-785. [6] Мохов О. И., Ферапонтов Е. В. // УМН. 1990. Т. 45. № 3. С. 191-192. [7] Ферапонтов Е. В. // Функц. анализ и его прилож. 1991. Т. 25. № 3. С. 37-49. [8] Mokhov O. I. // Phys. Lett. A. 1992. V. 166. № 3, 4. P. 215-216.

Центр нелинейных исследований

Принято редколлегией при Институте теоретической физики им. Л. Д. Ландау РАН, 11.05 .2000

Department of Mathematics, University of Paderborn, Germany

E-mail: mokhov@genesis.mi.ras.ru, mokhov@landau.ac.ru; mokhov@uni-paderborn.de 